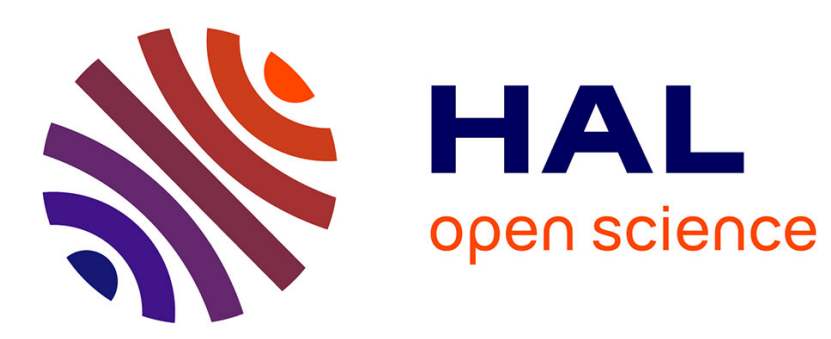

\title{
Theoretical Analysis of UNB-based IoT Networks with Path Loss and Random Spectrum Access
}

\author{
Yuqi Mo, Claire Goursaud, Jean-Marie Gorce
}

\section{To cite this version:}

Yuqi Mo, Claire Goursaud, Jean-Marie Gorce. Theoretical Analysis of UNB-based IoT Networks with Path Loss and Random Spectrum Access. 27th IEEE International Symposium on Personal, Indoor and Mobile Radio Communications (PIMRC), Sep 2016, Valencia, Spain. hal-01389365

\section{HAL Id: hal-01389365 \\ https://hal.inria.fr/hal-01389365}

Submitted on 28 Oct 2016

HAL is a multi-disciplinary open access archive for the deposit and dissemination of scientific research documents, whether they are published or not. The documents may come from teaching and research institutions in France or abroad, or from public or private research centers.
L'archive ouverte pluridisciplinaire HAL, est destinée au dépôt et à la diffusion de documents scientifiques de niveau recherche, publiés ou non, émanant des établissements d'enseignement et de recherche français ou étrangers, des laboratoires publics ou privés. 


\title{
Theoretical Analysis of UNB-based IoT Networks with Path Loss and Random Spectrum Access
}

\author{
Yuqi $\mathrm{MO}^{* \dagger}$, Claire GOURSAUD* and Jean-Marie GORCE* \\ ${ }^{*}$ CITI Labs, INSA-Lyon, Villeurbanne, France \\ ${ }^{\dagger}$ Sigfox Company, Building E-volution, Labège, France \\ Email: mo.yuqi@insa-lyon.fr; claire.goursaud@insa-lyon.fr; jean-marie.gorce@insa-lyon.fr;
}

\begin{abstract}
UNB is dedicated to long range and low power transmission in IoT networks. The channel access is RandomFTMA, where nodes select their time and frequency in a random and continuous way. This randomness leads to a new behavior of the interference which has not been theoretically analyzed yet, when considering the pathloss of nodes located randomly in an area. In this paper, in order to quantify the system performance, we derive and exploit a theoretical expression of the packet error rate in a UNB based IoT network, when taking into account both interference due to the spectral randomness and path loss due to the propagation.
\end{abstract}

\section{INTRODUCTION}

IoT (Internet of things) refer to networks of physical devices that have communications capabilities. It is expected that, by 2020 , there will be 20 billions of communicating objects in the world [1]. They will be designed to support many sorts of applications such as smart metering, smart vehicles, surveillance, etc. [2]. To do so, these objects collect and transfer information. One may note that most of these nodes have only a small amount of data to transfer. Thus, a single collecting point can serve, from a capacity point of view, a high number of nodes. For low density deployments, this permits a coverage of tens of kilometers. Nonetheless, the majority of devices are battery-based. As the battery maintenance is to be avoid due to the high number of nodes, a low energy consumption is also a strong requirement for IoT. Thus the challenges of IoT networks are to connect a high number of devices, to be able to manage a bursty access to the medium, to cover a wide area, while keeping it low cost and energy efficient.

To meet these specific demands of IoT networks, new technologies dedicated to LPWAN (Low Power Wide Area Network) have recently emerged on the market [3], such as: LoRa [4] (promoted by the LoRa Alliance), RPMA [5] (developed and exploited by Ingenu), and UNB (Ultra Narrow Band, developed and exploited by SigFox) [6]. Interestingly, two opposite approaches have been exploited to perform the targeted long range transmission. Spreading spectrum is considered in LoRa and RPMA, while UNB permits to transmit messages by using extreme narrow band signals $(100-200 \mathrm{~Hz}$, about one thousand times smaller than the whole channel bandwidth). Key advantages of UNB are its simplicity and its bigger practical coverage than the others [3]. We thus focus on UNB in this paper.
The natural channel access scheme of UNB is RandomFTMA (Random Frequency and Time Multiple Access). Every node can access the channel at their will, in an unslotted random way, both in time and in frequency domain [7]. On one hand, the advantage of this uncontrolled access scheme is that the channel reservation of transmission is saved. On the other hand, this randomness in spectrum and time does not prevent from potential interference. In particular, contrarily to traditionally defined transmissions where channelization can be performed, there can be partial spectral conflicts in UNB based networks. This specificity of the interference level has been detailed and modeled in [8], while its impact on the system performance has been estimated in [9]. However, these studies considered a perfect channel case.

In practice, the received signal is attenuated, especially for such long range transmissions. According to Shannon [11], path loss is the main contributor of the received signal power attenuation, which depends mainly on the distance between the transmitter and the receiver. For a network where numerous nodes are randomly located, stochastic geometry has been developed as an analytic tool to model the interference, and to quantify the network performance [12]. For example, stochastic geometry has been used to analyze systems such as simple random access in ad hoc networks (ALOHA) [13], dense IEEE 802.11 networks [14], multi-tier and cognitive cellular wireless networks [15], [16], [17].

All these works of stochastic geometry are interesting, because they provide us interference models for different kinds of networks. Nevertheless, as all these studies are based on channelization, they do not consider that interference could come from partial collisions in the frequency domain. A way to introduce this characteristic in the stochastic geometry model is to consider a marked spatial Poisson Point Process (PPP), where the mark models the residual proportion of interference which is perceived on the desired transmission. However, due to the shape of this rejection coefficient function, such approach leads to untractable expressions.

We present in this paper another way to compute the Packet Error Probability (PER). The main contribution is to provide an analytic expression of a UNB-based system performance, when considering both the path loss effect and the specific behavior of the interference in the spectral domain.

The rest of the paper is constructed as follows: in Section II we model the network and state all the hypothesis. In 


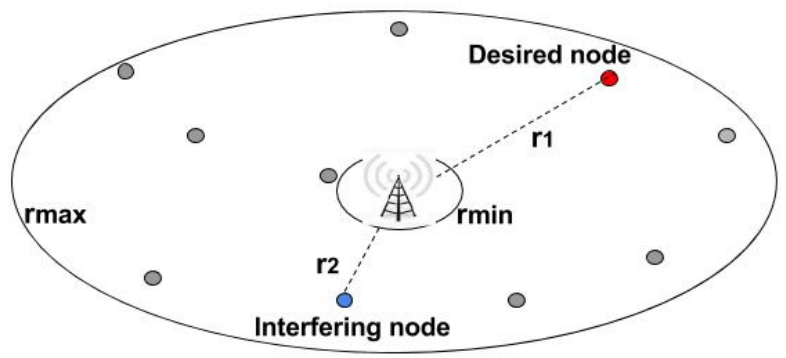

Fig. 1. Illustration of network topology: area range is $\left[r_{\min }, r_{\max }\right]$, red point is the desired node, at a distance of $r_{1}$; blue point is interfering node, at a distance of $r_{2}$

Section III, we derive theoretically the expression of packet error rate in UNB network. Then, in Section IV, we validate our analysis and present numerical results. Finally, Section V concludes this paper.

\section{Modeling AND ASSUMPTIONS}

\section{A. Network topology}

We consider a cellular topology, in which nodes only communicate with the Base Stations (BS). As the interference (so the performance) is linked to the BS range, for the sake of simplification, we focus on the behavior of a unique cell. The BS defines the cell center. We suppose that nodes are uniformly distributed in a disk form area, whose range is $\left[r_{\text {max }}, r_{\text {min }}\right]$, as shown in Fig. 1. Nodes are positioned randomly inside the cell, but no nodes are placed in the inner disk of the cell.

The BS is considered to always be in reception mode, and to scan the whole bandwidth for potential transmissions (as done in SigFox network). At any given moment, we denote by $N$ the number of active nodes actually transmitting. We assume that all these $N$ nodes emit with the same emission power. For each detected transmission (even simultaneous ones), the BS processes the incoming message(s).

We assume that transmissions are performed with the UNB modulation technique. Binary data are broadcast with a BPSK modulation at a very low rate (e.g. $R_{b}=100 \mathrm{bps}$ ). The transmitted signal thus occupies a very narrow band (e.g. about $b=100 \mathrm{~Hz}$ ). Transmissions are realized at a randomly chosen (in an unslotted way) carrier frequency in a much larger band $B$ (typically $192 \mathrm{kHz}$ ). The specificity of UNB comes from the inherent lack of precision of any oscillator. Indeed, offset always exists between the targeted frequency and the actual one. But, when the frequency uncertainty is higher than the signal bandwidth, the system is said to be UNB. In this case, contrarily to narrow band systems, it is not possible to obtain non-overlapping frequency channels with reasonable guard intervals [9]. This leads to a new paradigm for the multiple access scheme and the interference modeling, as channelization is not pertinent anymore for UNB.

As each node randomly chooses its transmission frequency, the uncontrolled medium access may lead to interference or packet collisions between the active users, especially when

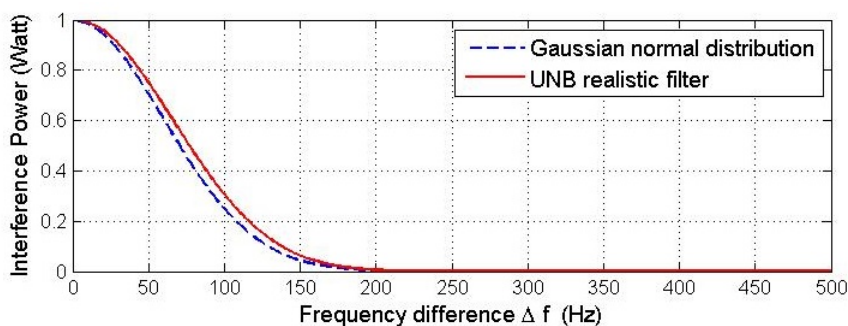

Fig. 2. Interference behavior as a function of the frequency spacing $\Delta f$ between desired node and interfering node

two or more nodes pick the same or close frequencies. This specific interference is described in the next section.

\section{B. Interference model}

Without loss of generality, we consider that node 1 is the desired node, while all the others are called interfering nodes as they can collide with the desired node.

The received signal at the base-station is the sum of all signals transmitted by each node in the active set $\mathcal{A}$ and can be expressed as:

$$
\begin{aligned}
r(t) & =h_{1}(t) * g\left(f_{1}, t\right) * s_{1}(t) \\
& +\sum_{y \in\{\mathcal{A}-1\}} h_{y}(t) * g\left(f_{y}, t\right) * s_{y}(t)+w(t)
\end{aligned}
$$

where, for any active node $i \in \mathcal{A}, s_{i}(t)$ is the BPSK symbols vector; $g\left(f_{i}, t\right)$ is the transmission FIR filter centered on the randomly chosen carrier frequency $f_{i} ; h_{i}(t)$ is the channel response; $*$ denotes the convolution operator, and $w(t)$ is an additive white Gaussian noise. As the signal is ultra narrow, the noise contribution is very low, and can be neglected compared to interference.

At the BS side, after filtering at the desired user carrier frequency $f_{1}$, the signal becomes:

$$
\begin{aligned}
r^{\prime}\left(f_{1}, t\right) & =g\left(f_{1}, t\right) * r(t) \\
& =h_{1} \cdot g\left(f_{1}, t\right) * g\left(f_{1}, t\right) * s_{1}(t) \\
& +\sum_{y \in\{\mathcal{A}-1\}} h_{y} \cdot g\left(f_{y}, t\right) * g\left(f_{1}, t\right) * s_{y}(t)
\end{aligned}
$$

We can first extract the received power corresponding to the signal of the desired node 1 :

$$
P_{s}=h_{1} \cdot \beta\left(f_{1}, f_{1}\right) \cdot P_{0}
$$

with $\beta\left(f_{1}, f_{1}\right)$ the filter auto correlation coefficient as it considers the transmission and reception filters centered at the same carrier frequency.

Similarly, the interference power $I_{y}$ caused by a single interferer on the desired signal follows:

$$
I_{y}=h_{y} \cdot \beta\left(f_{1}, f_{y}\right) \cdot P_{0}
$$

with $\beta\left(f_{1}, f_{y}\right)$ the rejection coefficient as the reception filter is centered on a different frequency than the transmission filter. This rejection coefficient quantifies the portion of emitted 
signal which is kept after filtering. It depends on the frequency spacing between the two carrier frequencies $\Delta f=\left|f_{1}-f_{y}\right|$ as presented in Fig. 2. We can observe that all the interference is kept $\left(\beta\left(f_{1}, f_{y}\right)=1\right)$ when $\Delta f=0$ while it tends to 0 as the interferer frequency moves away from the desired user one.

The exact expression of the rejection coefficient depends on the filter shape. When considering the filters used in SigFox transmissions, the interference power can be approximated by a zero-mean Gaussian function, depending on the frequency difference $\Delta f$ :

$$
\beta(\Delta f)=\frac{150}{\sigma \sqrt{2 \pi}} \exp \frac{-\Delta f^{2}}{2 \sigma^{2}}
$$

with $\sigma=60$ [8].

We will use this approximation to evaluate the interference contribution of each interfering node.

\section{THEORETICAL ANALYSIS}

In this section, we derive the theoretical expression of the PER (Packet Error Rate) when considering path loss and spectral interference. As we neglect the noise contribution compared to the interference, a packet is considered to be successfully transmitted if its SIR (Signal to Interference Ratio) is above a predefined threshold. We thus aim at expressing the PER as a function of following parameters: the whole available bandwidth $B$; the SIR threshold $S$ above which a packet is considered successful; the range of the considered cell $r_{\max }$, the exclusion radius $r_{\min }$; and the number of active nodes $N$.

Rigorously, packet loss can be due to a unique interferer, or the aggregation of several interferers. However, in practice, the second case is negligible compared to the first one. We thus consider the case where one node interrupts the desired one. So, we first consider the case of 2 active users before generalizing it to $N$ active users.

We first express the SIR in the 2 users case. We consider that the propagation model is free space, and packets from all nodes are sent at the same time, with the same emission power and antenna gain. We can thus express the received power of any transmitting node as a function of the node distance and the reference power $P_{0}$ observed at reference distance $r_{0}$. Distance between desired node (resp. interfering node) and BS is $r_{1}$ (resp. $r_{2}$ ), as illustrated in Fig. 1. Thus, the SIR of the desired node can be expressed as :

$$
S I R=\frac{P_{0}\left(\frac{r_{0}}{r_{1}}\right)^{2}}{P_{0}\left(\frac{r_{0}}{r_{2}}\right)^{2} \beta(\Delta f)}=\left(\frac{r_{2}}{r_{1}}\right)^{2} \frac{1}{\beta(\Delta f)}
$$

If SIR of a given packet is smaller than the threshold $S$, this packet is considered lost. We have $S I R \leq S$ when :

$$
r_{2} \leq r_{1} \sqrt{S \beta(\Delta f)}
$$

We decompose (7) by using the law of total probability by conditioning on $\Delta f$. Hence, the PER becomes :

$$
\begin{aligned}
P E R & =\int_{0}^{B} P(S I R \leq S \mid \Delta f) P(\Delta f) \mathrm{d} \Delta f \\
& =\int_{0}^{B} P\left(r_{2} \leq r_{\mathrm{I}} \sqrt{S \beta(\Delta f)} \mid \Delta f\right) P(\Delta f) \mathrm{d} \Delta f
\end{aligned}
$$

First of all, we derive $P(\Delta f)$. Desired and interfering nodes choose their frequencies $f_{1}$ and $f_{2}$ randomly and uniformly in $[0, B]$. Thus, their spectral difference $\Delta f$ follows the following probability distribution function:

$$
P(\Delta f)= \begin{cases}\frac{2}{B}\left(1-\frac{\Delta f}{B}\right) & \text { for } \Delta f \in[0, B] \\ 0 & \text { elsewhere }\end{cases}
$$

Secondly, we calculate $P\left(r_{2} \leq r_{1} \sqrt{S \beta(\Delta f)} \mid \Delta f\right)$. We further decompose it by conditioning on $\Delta f$ and $r_{1}$. So we obtain:

$$
\begin{aligned}
& P\left(r_{2} \leq r_{1} \sqrt{S \beta(\Delta f)} \mid \Delta f\right) \\
& =\int_{r_{\text {min }}}^{r_{\max }} P\left(r_{2} \leq r_{1} \sqrt{S \beta(\Delta f)} \mid \Delta f \cap r_{1}\right) P\left(r_{1}\right) \mathrm{d} r_{1}
\end{aligned}
$$

$P(r)$ presents the probability of choosing a random position (radius $r$ ) in a disk form area of $\left[r_{\min }, r_{\max }\right]$ :

$$
P(r)= \begin{cases}\frac{2 r}{r_{\max }^{2}-r_{\min }^{2}}=\frac{2 r}{k^{2}} & \text { for } r \in\left[r_{\min }, r_{\max }\right] \\ 0 & \text { elsewhere }\end{cases}
$$

with $k^{2}=r_{\max }^{2}-r_{\min }^{2}$.

$P\left(r_{2} \leq r_{1} \sqrt{S \beta(\Delta f)} \mid \Delta f \cap r_{1}\right)$ represents the probability that $r_{2}$ is smaller than one specific value, in the considered area. As $r_{2}$ is constrained to be in the range $\left[r_{\min }, r_{\max }\right], 3$ cases must be considered to correctly define the integration bounds, depending on the range value of $r_{1} \sqrt{S \beta(\Delta f)}$ :

$$
\begin{aligned}
& P\left(r_{2} \leq r_{1} \sqrt{S \beta(\Delta f)} \mid \Delta f \cap r_{1}\right) \\
& = \begin{cases}\int_{r_{\min }}^{r_{\min }} P\left(r_{2}\right) \mathrm{d} r_{2} & \text { if } r_{1} \sqrt{S \beta(\Delta f)} \leq r_{\text {min }}, \\
\int_{r_{\min }}^{r_{1} \sqrt{S \beta(\Delta f)}} P\left(r_{2}\right) \mathrm{d} r_{2} & \text { if } r_{\min } \leq r_{\mathrm{N}} \sqrt{S \beta(\Delta f)} \leq r_{\max } \\
\int_{r_{\min }}^{r_{\max }} P\left(r_{2}\right) \mathrm{d} r_{2} & \text { if } r_{1} \sqrt{S \beta(\Delta f)} \geq r_{\max }\end{cases} \\
& = \begin{cases}0 & \text { if } r_{1} \sqrt{S \beta(\Delta f)} \leq r_{\min }, \\
\frac{r_{1}^{2} S \beta(\Delta f)-r_{\min }^{2}}{k^{2}} & \text { if } r_{\min } \leq r_{1} \sqrt{S \beta(\Delta f)} \leq r_{\max } \\
1 & \text { if } r_{1} \sqrt{S \beta(\Delta f)} \geq r_{\max }\end{cases}
\end{aligned}
$$

The first line in (12) (resp third line) renders the fact that, the targeted range threshold is so small (resp. so high) that, in the targeted area, no node can be (resp. all the nodes are) closer. The second line is the intermediate case, and its value depends on the desired node location. 
With the knowledge of (11) and (12), we can compute $P\left(r_{2} \leq r_{1} \sqrt{S \beta(\Delta f)} \mid \Delta f\right)$ as defined in (10). However, as the conditions in (12) depends on both $r_{1}$ and $\Delta f$, the expression is computed differently depending on the range of $\Delta f$. We have distinguished 4 cases based on the value of $\sqrt{S \beta(\Delta f)}$ which characterizes the relative positioning of the desired and interfering node:

1) case 1: $\sqrt{S \beta(\Delta f)} \leq \frac{r_{\min }}{r_{\max }}$. This case is directly related to the first line in (12). Due to inequality transitivity, the $r_{1} \sqrt{S \beta(\Delta f)} \leq r_{\max } \sqrt{S \beta(\Delta f)} \leq r_{\min }$ condition and the (7) one imply that $r_{2} \leq r_{\text {min }}$. This is not achievable, as there is no node inside the exclusion area of radius $r_{\text {min }}$. Thus, for such $\Delta f$, it is not possible to find a node close enough to the BS to create sufficient interference. Thus, $P\left(r_{2} \leq r_{1} \sqrt{S \beta(\Delta f)} \mid \Delta f\right)$ is null.

2) case 2: $\frac{r_{\min }}{r_{\max }} \leq \sqrt{S \beta(\Delta f)} \leq 1$. This corresponds to the case where the interfering node must be closer to the receiver than the desired node (but not in the exclusion area). In this case, the left inequality in the second line in (12) is verified as $r_{\min } \leq r_{1} \sqrt{S \beta(\Delta f)}$ is equivalent to $\frac{r_{\min }}{r_{\text {max }}} \leq \frac{r_{\min }}{r_{1}} \leq \sqrt{S \beta(\Delta f)} \leq 1$. Hence, all desired nodes such that $r_{1} \in\left[\frac{r_{\min }}{\sqrt{S \beta(\Delta f)}}, r_{\max }\right]$ contribute to this case.

3) case 3: $1 \leq \sqrt{S \beta(\Delta f)} \leq \frac{r_{\max }}{r_{\min }}$. Contrarily to the second case, the interfering node can be further from the receiver than the desired node. In this case, nodes with a distance $r_{1} \in\left[r_{\min }, \frac{r_{\max }}{\sqrt{S \beta(\Delta f)}}\right]$ verify the right inequality in the second line of (12), while those such that $r_{1} \in\left[\frac{r_{\max }}{\sqrt{S \beta(\Delta f)}}, r_{\max }\right]$ verify the third line.

4) case 4: $\sqrt{S \beta(\Delta f)} \geq \frac{r_{\max }}{r_{\min }}$. In this last case, even the closest desired user is interfered by any node in the cell. This is directly related to the third line in (12), which leads to $\frac{r_{2}}{r_{1}} \leq$ $\frac{r_{\max }}{r_{\min }} \leq \sqrt{S \beta(\Delta f)}$. So $P\left(r_{2} \leq r_{1} \sqrt{S \beta(\Delta f)} \mid \Delta f\right)=1$.

Accordingly, we can compute the integral of all the four cases:

$$
\begin{aligned}
& P\left(r_{2} \leq r_{1} \sqrt{S \beta(\Delta f)} \mid \Delta f\right) \\
& =\left\{\begin{array}{l}
0 \\
\int_{\frac{r_{\min }}{\sqrt{S \beta(\Delta f)}}}^{r_{\max }} \frac{r_{1}^{2} S \beta(\Delta f)-r_{\text {min }}{ }^{2}}{k^{2}} \frac{2 r_{1}}{k^{2}} \mathrm{~d} r_{1} \\
\int_{r_{\min }}^{\frac{r_{\text {max }}}{\sqrt{S \beta(\Delta f)}}} \frac{r_{1}{ }^{2} S \beta(\Delta f)-r_{\text {min }}{ }^{2}}{k^{2}} \frac{2 r_{1}}{k^{2}} \mathrm{~d} r_{1} \\
+\int_{\frac{r_{\max }}{\sqrt{S \beta(\Delta f)}}}^{r_{\max }} \\
1
\end{array}\right. \\
& = \begin{cases}0 & \text { if } \sqrt{S \beta(\Delta f)} \leq \frac{r_{\min }}{r_{\max }}, \\
\frac{a}{S \beta(\Delta f)}+b S \beta(\Delta f)+c & \text { if } \frac{r_{\min }}{r_{\max }} \leq \sqrt{S \beta(\Delta f)} \leq 1 \\
\frac{d}{S \beta(\Delta f)}+e S \beta(\Delta f)+f & \text { if } 1 \leq \sqrt{S \beta(\Delta f)} \leq \frac{r_{\max }}{r_{\min }} \\
1 & \text { if } \sqrt{S \beta(\Delta f)} \geq \frac{r_{\max }}{r_{\min }}\end{cases}
\end{aligned}
$$$$
\text { if } \sqrt{S \beta(\Delta f)} \leq \frac{r_{\min }}{r_{\max }},
$$$$
\text { if } \frac{r_{\min }}{r_{\max }} \leq \sqrt{S \beta(\Delta f)} \leq 1
$$

if $1 \leq \sqrt{S \beta(\Delta f)} \leq \frac{r_{\max }}{r_{\min }}$ if $\sqrt{S \beta(\Delta f)} \geq \frac{r_{\max }}{r_{\text {min }}}$

\section{A. Validation}

In this section, we present simulation results based on realistic network configurations (based on SigFox one). We compare

with the following constants :

$$
\begin{aligned}
& a=\frac{r_{\min }{ }^{4}}{2 k^{4}}, \quad d=\frac{r_{\max }^{4}}{2 k^{4}}-\frac{r_{\min }^{2} r_{\max }^{2}}{k^{4}}-\frac{r_{\max }^{2}}{k^{2}} \\
& b=\frac{r_{\max }{ }^{4}}{2 k^{4}} \\
& c=-\frac{r_{\max }^{2} r_{\min }{ }^{2}}{k^{4}}, \\
& e=-\frac{r_{\min }^{4}}{2 k^{4}} \\
& f=\frac{r_{\min }^{4}}{k^{4}}+\frac{r_{\max }^{2}}{k^{2}}
\end{aligned}
$$

With the above expressions, we can finally derive (9). We first express the integral bounds according to the cases defined in (14). First, $\sqrt{S \beta(\Delta f)} \leq \frac{r_{\min }}{r_{\max }}$ is equivalent to $\beta^{-1}\left(\left(\frac{r_{\min }}{r_{\max }}\right)^{2} \frac{1}{S}\right) \leq \Delta f$. As the maximum value of $\Delta f$ is $B$, the lower bound must be constrained by $B$. Thus, the integral bounds for this case are: $b_{1}=$ $\min \left(\beta^{-1}\left(\left(\frac{r_{\max }}{r_{\min }}\right)^{2} \frac{1}{S}\right), B\right)$ and $b_{0}=B$.

Secondly, the same principle applies to $\frac{r_{\min }}{r_{\max }} \leq$ $\sqrt{S \beta(\Delta f)} \leq 1$, which leads to $\beta^{-1}\left(\frac{1}{S}\right) \leq \Delta f \leq$ $\beta^{-1}\left(\left(\frac{r_{\min }}{r_{\max }}\right)^{2} \frac{1}{S}\right)$. The integral bounds for this part are: $b_{1}$ and $b_{2}=\min \left(\beta^{-1}\left(\frac{1}{S}\right), B\right)$. Similarly, for $1 \leq$ $\sqrt{S \beta(\Delta f)} \leq \frac{r_{\max }}{r_{\min }}$, leads to $\beta^{-1}\left(\left(\frac{r_{\max }}{r_{\min }}\right)^{2} \frac{1}{S}\right) \leq \Delta f \leq$ $\beta^{-1}\left(\frac{1}{S}\right)$. The integral bounds are thus: $b_{2}$ and $b_{3}=$ $\min \left(\beta^{-1}\left(\left(\frac{r_{\max }}{r_{\min }}\right)^{2} \frac{1}{S}\right), B\right)$. Finally, $\sqrt{S \beta(\Delta f)} \geq \frac{r_{\max }}{r_{\min }}$ is equivalent to $0 \leq \Delta f \leq \beta^{-1}\left(\left(\frac{r_{\max }}{r_{\min }}\right)^{2} \frac{1}{S}\right)$. Thus $b_{4}=0$. One may note that $\beta(\Delta f) \in[0,1]$. Thus, $b_{3}$ exists only if $\left(\frac{r_{\max }}{r_{\min }}\right)^{2} \leq S$. If not, $b_{3}$ would be null, and the last term of integral would be null too.

As a consequence, the integral of (9) can be expressed as :

$$
\begin{aligned}
P E R= & \int_{0}^{B} P\left(r_{2} \leq r_{1} \sqrt{S \beta(\Delta f)} \mid \Delta f\right) P(\Delta f) \mathrm{d} \Delta f \\
= & +\int_{b_{2}}^{b_{1}}\left(\frac{a}{S I R_{t h} \beta(\Delta f)}+b S I R_{t h} \beta(\Delta f)+c\right) P(\Delta f) \mathrm{d} \Delta f \\
& +\int_{b_{3}}^{b_{2}}\left(\frac{d}{S I R_{t h} \beta(\Delta f)}+e S I R_{t h} \beta(\Delta f)+f\right) P(\Delta f) \mathrm{d} \Delta f \\
& +\int_{b_{4}}^{b_{3}} 1 * P(\Delta f) \mathrm{d} \Delta f
\end{aligned}
$$

The final expression of this PER is given in (16).

It represents the PER when there are 2 active nodes. We now extend it to $N$ active nodes. When there are $N$ active nodes, any of the $N-1$ nodes (i.e. all nodes except the desired node) can be an interfering node. Accordingly, the success probability of the desired node, is that it will not be interrupted by any of $N-1$ nodes. Consequently, the packet error rate is given by:

$$
P E R_{(N)}=1-(1-P E R)^{N-1}
$$

\section{VALIDATION AND NUMERICAL RESUlts}




$$
\begin{aligned}
& P E R=\frac{2}{B}\left[\frac{a \sigma^{2} \pi}{S 150} \operatorname{erfi}\left(\frac{\Delta f}{\sqrt{2 \sigma^{2}}}\right)+75 b S \operatorname{erf}\left(\frac{\Delta f}{\sqrt{2 \sigma^{2}}}\right)+c \Delta f-\frac{a \sigma^{3} \sqrt{2 \pi}}{B S 150} \exp \frac{\Delta f^{2}}{2 \sigma^{2}}+\frac{b S 150 \sigma}{B \sqrt{2 \pi} \exp \frac{\Delta f^{2}}{2 \sigma^{2}}}-\frac{c(\Delta f)^{2}}{2 B}\right]_{b_{2}}^{b_{1}} \\
& +\frac{2}{B}\left[\frac{d \sigma^{2} \pi}{S 150} \operatorname{erfi}\left(\frac{\Delta f}{\sqrt{2 \sigma^{2}}}\right)+75 e S \operatorname{erf}\left(\frac{\Delta f}{\sqrt{2 \sigma^{2}}}\right)+f \Delta f-\frac{d \sigma^{3} \sqrt{2 \pi}}{B S 150} \exp \frac{\Delta f^{2}}{2 \sigma^{2}}+\frac{e S 150 \sigma}{B \sqrt{2 \pi} \exp \frac{\Delta f^{2}}{2 \sigma^{2}}}-\frac{f(\Delta f)^{2}}{2 B}\right]_{b_{3}}^{b_{2}}+\frac{2}{B}\left[\Delta f-\frac{\Delta f^{2}}{2 B}\right]_{b_{4}}^{b_{3}}
\end{aligned}
$$

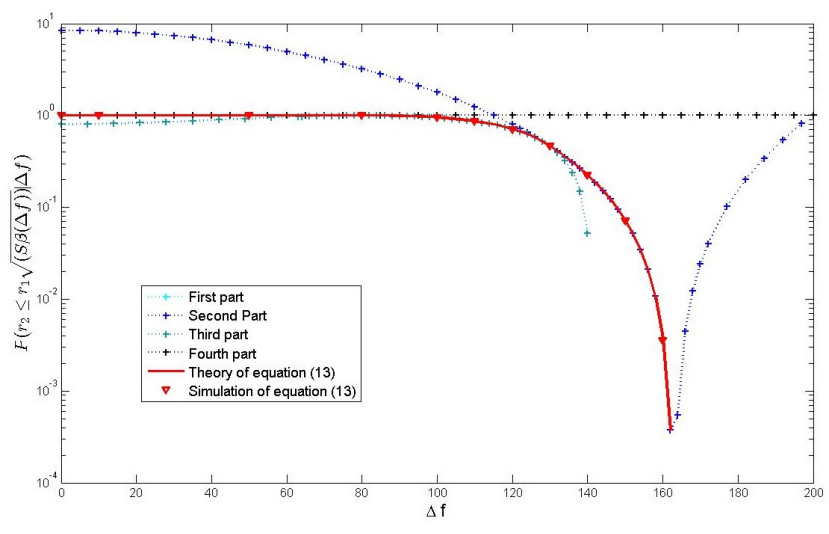

Fig. 3. Segmentation of the four parts of $P\left(r_{2} \leq r_{1} \sqrt{S \beta(\Delta f)} \mid \Delta f\right)$ in (14), with $S N R t h=10 \mathrm{~dB}, B W=12000 \mathrm{~Hz}, r_{\min }=30 \mathrm{~m}$, and $r_{\max }=60 \mathrm{~m}$.

them to our theoretical values, to validate the expression of $\operatorname{PER}_{(N)}$ (17). We consider the following key parameters: number of active nodes $N$, bandwidth $B$, range of transmission area $\left[r_{\min }, r_{\max }\right]$, and the threshold of signal-interferenceratio $S$. For a better understanding, we provide here the unit of $S$ in $\mathrm{dB}$. But in the theoretical expression, the linear value of $S$ must be used $\left(10^{\frac{S}{10}}\right)$.

The simulation results are obtained with the discrete-event simulator WSNet [18].

We first provide an intermediate validation of our analysis, by comparing (14) with simulation behavior in Fig.3. We can observe the four parts of the expression (in blue), which define in turn the final expression (red curve). Besides, we can verify that the simulation values (red triangle) are well predicted by the theory.

To finalize this validation, we present on Fig. 4-6, the evolution of $P E R_{(N)}$ by varying $B$, and along with another different parameter for each figure. From a global point of view, we can observe that simulation results match well with theoretical ones. Therefore, we can deduce that the formula (17) is validated.

\section{B. Performances analysis}

We now analyze these figures more precisely. In Fig. 4, the area ranges are set to $[30,1000]$. We can see that PER decreases when $B$ increases. Indeed, as there are more available transmission resources, there are less risks that nodes would collide. In addition, PER increases with $N$. When numerous

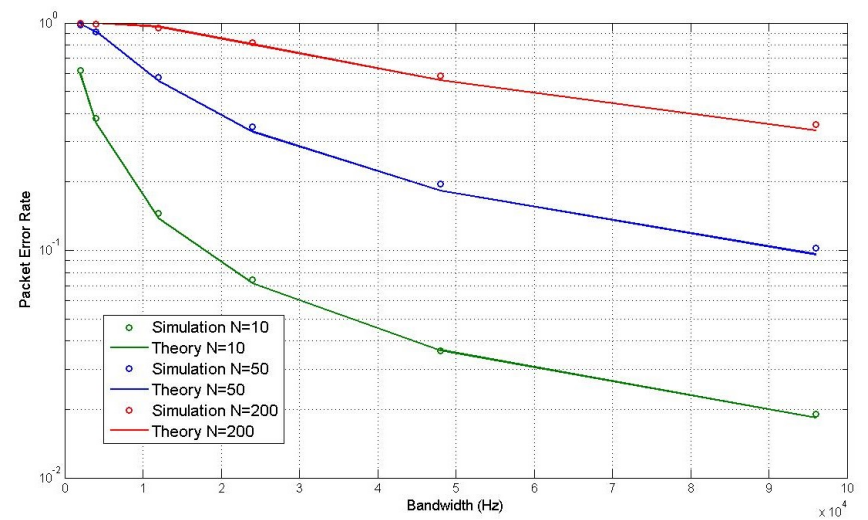

Fig. 4. PER vs bandwidth $B$, with different number of active nodes $N$, $r_{\max }=1000 \mathrm{~m}, r_{\min }=30 \mathrm{~m}, S=6.8 \mathrm{~dB}$.

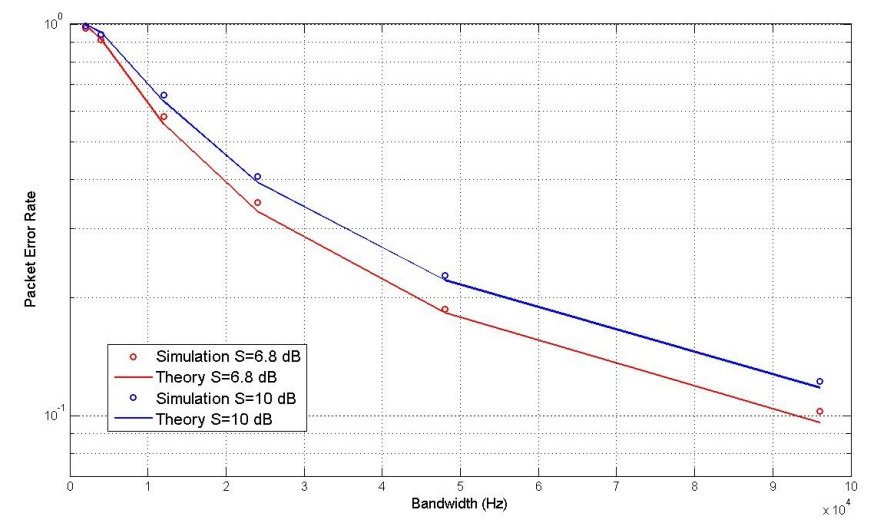

Fig. 5. PER vs bandwidth $B$, with different signal-interference-ratio threshold $S \mathrm{~dB}, N=50, r_{\max }=1000 \mathrm{~m}, r_{\min }=30 \mathrm{~m}$.

actives nodes transmit at the same time, the chance that their frequencies fall into the interfering zone gets higher.

Furthermore, we verify that PER increases when the threshold $S$ becomes bigger, as shown in Fig. 5. When the success criteria becomes stricter, nodes have less chances to attain $S$, so the network performance becomes worse. Fig. 6 shows that when the maximum range $r_{\max }$ changes from 60 to 1000, we have a little bit more errors. Meanwhile, when $r_{\max }$ changes from 1000 to $10^{6}$, their PER evolution totally overlap. It indicates that, when the area is already large, the range does not have many impact on network performance.

To sum up, the usual performance statements are verified: more network load induces more errors, such as small bandwidth and too many nodes; a more strict SIR threshold leads 


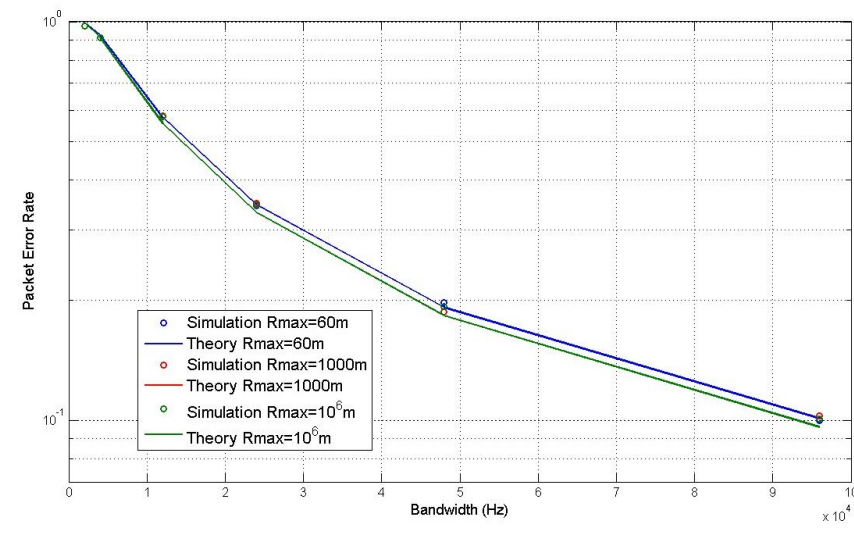

Fig. 6. PER vs bandwidth $B$, with different maximum range of area $r_{\max }$, $N=50, r_{\min }=30 \mathrm{~m}, S=6.8 \mathrm{~dB}$.

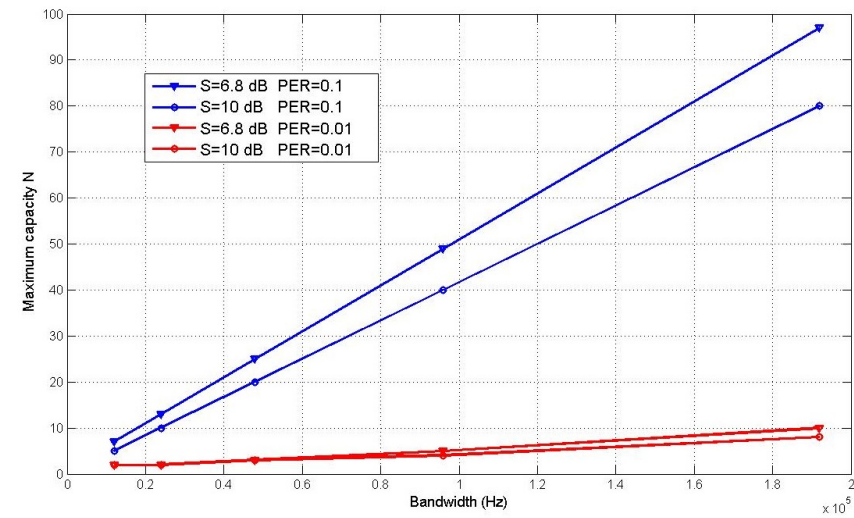

Fig. 7. Maximum capacity $N$ vs bandwidth $B$, with different signalinterference-ratio threshold $S \mathrm{~dB}$, and PER, $r_{\min }=30 \mathrm{~m}, r_{\max }=60$ $\mathrm{m}$.

to a higher PER; while the influence of area range to network performance depends on the scale.

\section{Results Exploitation}

In this section, we exploit the theoretical expression of PER to find out the maximum capacity. This parameter is defined in this study by the maximum number of active nodes $N$ that the network can support simultaneously, while maintaining a targeted PER. We have reported on Fig. 7, the capacity when the PER is targeted to be smaller than 0.1. We can observe that with a larger bandwidth, the system can contain more nodes; nevertheless, with a stricter signal interference ratio threshold, less active nodes can be served. More importantly, we can observe that the capacity increases linearly with the available bandwidth. It is thus easy to scale the bandwidth in accordance to the number of nodes to serve, when keeping the same targeted SIR. However, the theoretical expression still provides an efficient tool for any SIR.

\section{CONCLUSION}

In this paper, we have theoretically evaluated the packet error rate of a UNB based IoT network, by considering both interference due to spectral randomness, and pathloss. We have derived the expression of PER and validated it by simulations based on realistic configurations. We have evaluated the network performance when varying several parameters: active nodes $N$, bandwidth $B$, threshold of signal-interference-ratio $S$, and the range of transmission area $\left[r_{\min }, r_{\max }\right]$. One may note that this analysis can be the theoretical basis for further studies such as the interference cancellation.

\section{ACKNOWLEDGMENT}

The authors would like to thank Christophe Fourtet (Sigfox company) for providing realistic UNB network parameters in this study.

\section{REFERENCES}

[1] Jayavardhana Gubbi, Rajkumar Buyya, Slaven Marusic, Marimuthu Palaniswami, Internet of Things (IoT): A vision, architectural elements, and future directions, Future Generation Computer Systems, Volume 29, Issue 7, September 2013, Pages 1645-1660.

[2] L. Da Xu, W. He, S. Li, "Internet of Things in industries: A survey" in IEEE Transactions on Industrial Informatics, 10(4), 2014, pp2233-2243.

[3] C. Goursaud and J. M. Gorce, "Dedicated networks for IoT: PHY / MAC state of the art and challenges", EAI Endorsed Transactions on Internet of Things, vol. 15, no. 1, October 2015

[4] http://lora-alliance.org/What-Is-LoRa/Technology (accessed 2016/04/04)

[5] http://www.weightless.org/ (accessed 2016/01/04)

[6] http://www.sigfox.com/en/\#!/technology (accessed 2016/04/04)

[7] C. Goursaud, and Y. Mo "Random Unslotted Time-Frequency ALOHA: Theory and Application to IoT UNB Networks," in 23^rd International Conference on Telecommunications (ICT), 2016, 16-18 May 2016.

[8] M.-T. Do, C. Goursaud, and J.-M. Gorce, "Interference modelling and analysis of random fdma schemes in ultra narrow band networks," in The Tenth Advanced International Conference on Telecommunications, AICT 2014, pp. 132-137, July 2014.

[9] M.-T. Do, C. Goursaud, and J.-M. Gorce, "On the benefits of RandomFDMA schemes in ultra narrow band networks," in 2014 12th International Symposium on Modeling and Optimization in Mobile, Ad Hoc, and Wireless Networks (WiOpt), pp. 672-677, 12-16 May 2014.

[10] Cardieri, Paulo, "Modeling interference in wireless ad hoc networks,"Communications Surveys \& Tutorials, IEEE, vol. 12, no. 4, pp. 551-572, 2010

[11] C. Shannon, A Mathematical Theory of Communication, Bell System Technical Journal, vol. 27, pp. 379423, 623656, Oct. 1948.

[12] M. Haenggi, J. G. Andrews, F. Baccelli, O. Dousse and M. Franceschetti, "Stochastic geometry and random graphs for the analysis and design of wireless networks," in IEEE Journal on Selected Areas in Communications, vol. 27, no. 7, pp. 1029-1046, September 2009.

[13] F. Baccelli, B. Blaszczyszyn and P. Muhlethaler, "An Aloha protocol for multihop mobile wireless networks," in IEEE Transactions on Information Theory, vol. 52, no. 2, pp. 421-436, Feb. 2006.

[14] H. Q. Nguyen, F. Baccelli and D. Kofman, "A Stochastic Geometry Analysis of Dense IEEE 802.11 Networks," IEEE INFOCOM 2007 - 26th IEEE International Conference on Computer Communications, Anchorage, AK, 2007, pp. 1199-1207.

[15] C. h. Lee and M. Haenggi, "Interference and Outage in Poisson Cognitive Networks," in IEEE Transactions on Wireless Communications, vol. 11, no. 4, pp. 1392-1401, April 2012.

[16] A. H. Sakr and E. Hossain, "Cognitive and Energy Harvesting-Based D2D Communication in Cellular Networks: Stochastic Geometry Modeling and Analysis," in IEEE Transactions on Communications, vol. 63, no. 5, pp. 1867-1880, May 2015.

[17] H. ElSawy, E. Hossain and M. Haenggi, "Stochastic Geometry for Modeling, Analysis, and Design of Multi-Tier and Cognitive Cellular Wireless Networks: A Survey," in IEEE Communications Surveys Tutorials, vol. 15, no. 3, pp. 996-1019, Third Quarter 2013.

[18] G. Chelius, A. Fraboulet \& E. Ben Hamida. http ://wsnet.gforge.inria.fr/, (accessed 2016/04/04). 